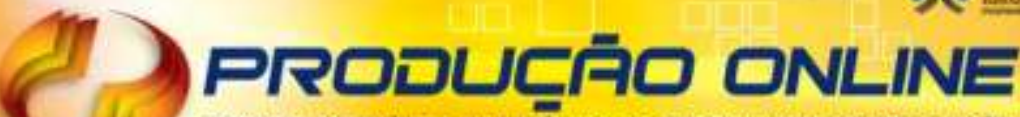 REVISTA CIENTIFICA ELETRÖNICA DE ENGENHARIA DE PRODUÇAO ISSN 1676-190|
}

\section{AVALIAÇÃO DA SATISFAÇÃO DOS USUÁRIOS DE SISTEMAS ERP NAS MÉDIAS EMPRESAS DE CONFECÇÃO DO VESTUÁRIO DE FORTALEZA}

\section{EVALUATION OF SATISFACTION OF USERS OF ERP SYSTEMS IN MEDIUM CLOTHING CONFECTION COMPANIES IN FORTALEZA}

\author{
Francismilton Teles* E-mail: francismilton@ig.com.br \\ Ricardo Moreira da Silva* E-mail: ricardomoreira0203@hotmail.com \\ *Universidade Federal da Paraíba (UFPB), João Pessoa, PB
}

\begin{abstract}
Resumo: Uma das ferramentas que colaboram para o aumento da competitividade nas pequenas e médias empresas é o uso de sistemas ERP (Enterprise Resources Planning), também conhecida como Sistema Integrado de Gestão, a qual quando bem utilizada, auxilia no processo de planejamento, gestão e controle das empresas. É muito importante a verificação do sucesso de utilização destes sistemas nas empresas e um dos meios mais aceitos de analisá-los é por meio da avaliação da satisfação dos seus usuários. Esta pesquisa avalia a satisfação dos usuários de sistemas ERP's nas médias empresas de confecção do vestuário no município de Fortaleza, um dos maiores pólos de confecção do Brasil. O método utilizado foi a pesquisa Survey, através da qual foram entrevistados 117 usuários de 25 empresas e seguiu o modelo de avaliação customizado por Ely (2010), onde são avaliados de forma quantitativa a satisfação dos usuários finais e usuárioschave de um sistema ERP. Os resultados apresentam, de forma geral, que os usuários estão satisfeitos com os sistemas ERP implantados. Também foi verificado por meio de análise multivariada, que o modelo utilizado foi adequado ao contexto das médias empresas, pois os testes estatísticos (Alfa de Cronbach, esfericidade de Bartlett e KMO) obtiveram valores bastante confiáveis.
\end{abstract}

Palavras-chave: Sistema ERP. Satisfação do Usuário. Tecnologia da Informação (TI).

Abstract: One of the tools that help increase the competitiveness in small and medium companies is the use of ERP (Enterprise Resources Planning), which, when used properly, assist in planning, management and control. It is very important to check the success of such systems in companies and one of the most widely accepted means of analyzing them is by evaluating the satisfaction of its users. This paper assess the satisfaction of users of ERP systems in medium manufacturing clothing in Fortaleza, one of the largest centers of clothing confection from Brazil. The method used was the Survey Research, though which 117 users were interviewed in 25 companies and followed the evaluation model customized by Ely (2010), which are evaluated quantitatively the satisfaction of end users and key users of an ERP system. The results show, in general, users are satisfied with the ERP systems implemented. It was also verified by multivariate analysis, the model used was appropriate to the context of medium enterprises, because the statistical tests (Cronbach's alpha, Bartlett's Sphericity and $\mathrm{KMO}$ ) scores were very reliable.

Keywords: ERP systems. User Satisfaction. Information Technology (TI).

\section{INTRODUÇÃO}

Um setor de bastante destaque no cenário nacional, tanto para as Pequenas e Médias Empresas (PME) como para as grandes empresas, é a cadeia têxtil e de

Revista Produção Online, Florianópolis, SC, v.14, n. 2, p. 533-559, abr./jun. 2014. 
confecção, a qual se inicia na agropecuária (fibras naturais) ou na indústria química (fibras manufaturadas), passando pela produção dos fios, tecidos, pelo beneficiamento e confecção, terminando no consumidor final (BRASIL, 2011). A presente pesquisa concentra-se no sub-segmento de confecção de vestuário.

Esta cadeia é responsável por 4,9\% do Produto Interno Bruto Brasileiro (PIB), representando bem mais do que outros segmentos. Este percentual coloca o setor em $7^{\circ}$ lugar no ranking das indústrias de transformação, em um total de 24 setores analisados (DINIZ FILHO, 2011).

A cadeia têxtil e de confecções possui a característica de formação de pólos regionais de produção. Os principais pólos brasileiros estão localizados em São Paulo, Rio de Janeiro, Santa Catarina e Ceará (COSTA e ROCHA, 2009). A presente pesquisa procurou abordar o pólo do Estado do Ceará, mais precisamente sua capital, a cidade de Fortaleza.

Grande parte da importância da indústria têxtil e de confecções no Ceará deve-se à tendência de deslocamento regional das grandes empresas de outras regiões para o estado cearense, estimuladas por incentivos fiscais e de infraestrutura, fornecidos pelo governo estadual, além da existência de mão-de-obra mais barata (COSTA e ROCHA, 2009).

Segundo Lemos et al. (2011), em paralelo com as grandes empresas de confecção do vestuário, o Ceará abriga um grande volume de micro e pequenas empresas, sendo estas umas das maiores fontes de emprego no Brasil, gerando, somente no Ceará, aproximadamente 37 mil empregos formais, fora os informais que são em maior número que os formais.

Apesar das PME's serem de fundamental importância para 0 desenvolvimento de uma região, como no caso da região de Fortaleza, elas enfrentam muitos desafios, principalmente no âmbito governamental.

Em uma pesquisa realizada pela Deloitte Consulting (2010), com 253 PME's, foi verificado que $91 \%$ das empresas pesquisadas consideram as altas cargas tributárias como fator de empecilho ao crescimento. Outros fatores citados foram: atração e retenção de pessoal qualificado, legislação trabalhista, dentre outros. A Figura 1 apresenta as principais dificuldades destacadas pela pesquisa nas PME's brasileiras.

Revista Produção Online, Florianópolis, SC, v.14, n. 2, p. 533-559, abr./jun. 2014. 
Figura 1 - Fatores que Dificultam o Desenvolvimento dos Negócios das PME’s (\%)

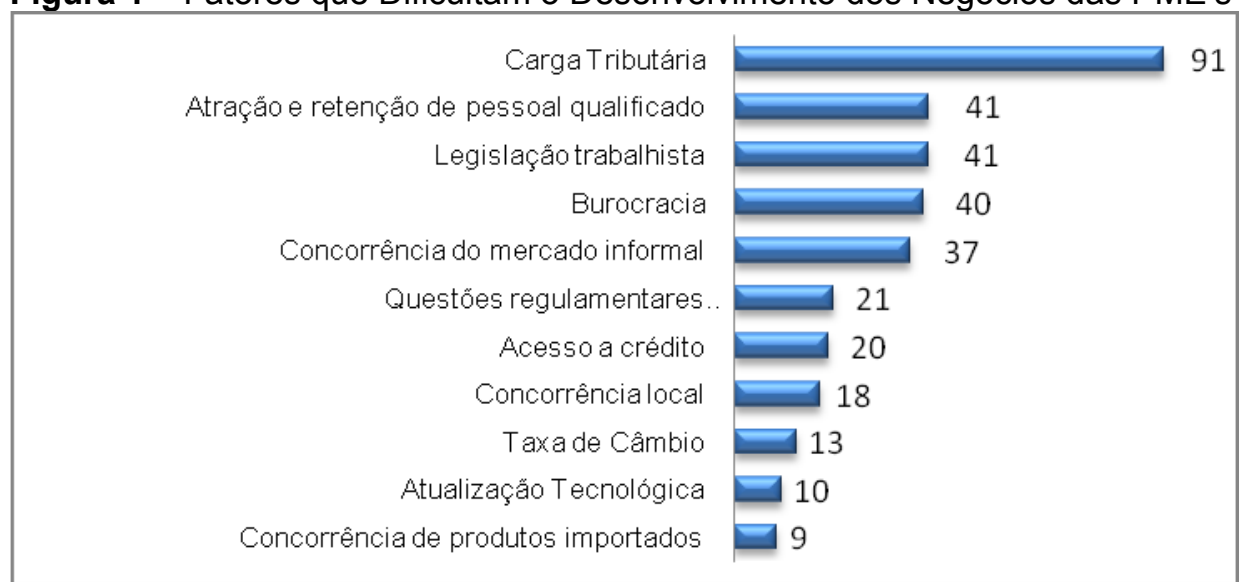

Fonte: Deloitte Consulting (2010)

Observa-se ainda na Figura 1 que a atualização tecnológica foi destacada como empecilho ao desenvolvimento por apenas $10 \%$ das empresas pesquisadas, mostrando que cada vez mais as inovações tecnológicas estão ao alcance das PME's. Ainda com relação à tecnologia, o mesmo estudo apontou que $43 \%$ das PME`s consideram de extrema relevância investir em tecnologia para garantir o avanço dos seus negócios. Dentre as tecnologias mais cobiçadas pelas empresas brasileiras destacam-se os sistemas de informação e, mais especificamente, os sistemas integrados de gestão, também chamados de sistemas ERP's (Enterprise Resource Planning).

Um estudo realizado pela International Data Corporation (IDC Brasil) (2009), empresa de consultoria em Tecnologia de Informação (TI), revelou que as vendas de sistemas integrados de gestão no Brasil superaram $R \$ 1,1$ bilhão no primeiro semestre de 2009, em comparação com os $\mathrm{R} \$ 923,6$ milhões do mesmo período em 2008. A estimativa para todo o ano de 2009 foi de que as empresas brasileiras tenham investido mais de 2,5 bilhões de reais em sistemas de ERP's, um crescimento de $17 \%$ em comparação a 2008.

Deste total de investimentos realizados no Brasil, uma boa parcela está voltada para as empresas de pequeno e médio porte. Segundo um estudo estatístico do SEBRAE, divulgado no ano de 2005, já havia, dentre as principais estratégias dos fornecedores de ERP, uma conquista de 140 mil empresas de pequeno porte com 20 a 99 funcionários (SEBRAE 2005 apud PINTO NETO, 2007).

Para manter sua sobrevivência as PME's precisam assegurar o controle de suas operações diante das atuais pressões que enfrentam, não só para atenderem à Revista Produção Online, Florianópolis, SC, v.14, n. 2, p. 533-559, abr./jun. 2014. 
demanda de mercado que vem aumentando, mas também para atenderem ao atual nível de confiança dos clientes. Alguns autores compreendem que a adoção de ERP's acrescenta uma grande vantagem competitiva, atendendo as expectativas dos clientes por prazos de entrega mais curtos, maior agilidade, qualidade e redução de custos (ENNS, 2001; MILTENBURG, 2001; KOH; SAAD, 2003; MALHOTRAA; TEMPONIB, 2010).

Devido ao grande impacto que um Sistema de Informação (SI) pode causar em uma organização é muito importante para elas conhecerem o sucesso de implantação de uma nova tecnologia. Porém, a capacidade de uma organização para avaliar a criação de valor e retorno econômico de um sistema ERP é uma tarefa difícil. Estas questões são particularmente importantes nas pequenas e médias empresas, onde a implementação de ERP e avaliação dos potenciais benefícios são ainda mais incertas (DAVENPORT, 1998; LEVY; POWELL, 2000 apud MALHOTRAA; TEMPONIB, 2010).

Estudos apontam que o sucesso de um SI pode ser avaliado através da satisfação dos seus usuários (IVES; OLSON; BAROUD, 1983; DOLL; TORKZADEH, 1988; WU; WANG, 2007; OLIVEIRA; CUNHA; SOUZA, 2008; ELY, 2010). Uma maneira imprescindível para se atingir o sucesso na implantação de tecnologias da informação é através da obtenção da satisfação dos usuários (ELY, 2010).

Diante das questões expostas neste trabalho sobre a importância das PME, do impacto dos ERP's e da necessidade de avaliação desses sistemas, surgiu o interesse de se realizar uma pesquisa em médias empresas da indústria de confecção do vestuário de Fortaleza para avaliação da satisfação dos usuários, sendo escolhido o modelo customizado por Ely (2010) de avaliação quantitativa dos usuários finais e usuários chave dos sistemas ERP.

Portanto, esta pesquisa usa como cenário a cidade de Fortaleza, capital do Estado do Ceará, nas empresas de médio porte da indústria de confecção do vestuário e a questão que segmentou este trabalho foi: qual o nível de satisfação dos usuários de sistemas ERP's nas médias empresas da indústria de confecção do vestuário em Fortaleza? 
Deste modo, o objetivo da pesquisa é Avaliar a satisfação dos usuários de Sistemas Integrados de Gestão (ERP) em Médias Empresas (ME) de confecção do vestuário do município de Fortaleza.

\section{FUNDAMENTAÇÃO TEÓRICA}

\subsection{Sistemas de Informação}

Segundo O’Brien (2004, p. 3) “[...] os Sistemas e Tecnologias de Informação tornaram-se componentes vitais quando se pretende alcançar o sucesso de empresas e organizações" e, por essa razão, constituem uma área extremamente importante nas organizações.

Pelas palavras de Stair e Reynolds (2006, p. 4) a definição de sistema de informação é descrita como "um conjunto de componentes inter-relacionados que coletam, manipulam e disseminam dados e informações para proporcionar um mecanismo de realimentação para atingir um objetivo". Os sistemas de informações devem apoiar as estratégias, os processos empresariais, as estruturas e a cultura organizacional a fim de aumentar o valor dos negócios em um ambiente dinâmico (SUSSMANN, 2005).

Laudon e Laudon (2004) completam dizendo que conhecer sistemas de informação é essencial, porque a maioria das organizações precisa deles para sobreviver e prosperar. No mesmo pensamento os autores destacam que esses sistemas podem auxiliar as empresas a estender seu alcance a locais distantes, oferecer novos produtos e serviços, reorganizar fluxos de tarefas e trabalho e, talvez, transformar radicalmente o modo como conduzem os negócios.

\subsection{Definições e conceitos de ERP}

Os sistemas ERP's possuem diversas definições na literatura devido às muitas características que eles apresentam. Uma das definições mais completas é expressa por Souza (2000) que define os sistemas ERP's como sendo sistemas integrados em forma de pacote de software comercial, com a finalidade de dar suporte à maioria das operações de uma empresa. São geralmente divididos em Revista Produção Online, Florianópolis, SC, v.14, n. 2, p. 533-559, abr./jun. 2014. 
módulos que se comunicam e utilizam uma mesma base de dados central, de modo que as informações alimentadas em um módulo são instantaneamente disponibilizadas para os demais módulos, permitem, também, a utilização de ferramentas de planejamento que podem analisar o impacto de decisões de manufatura, suprimentos, finanças ou recursos humanos em toda a empresa.

Corrêa, Gianesi e Caon (2001. p. 392) possuem uma visão mais detalhada de sua abrangência, destacando que o ERP:

[...] é basicamente composto de módulos que atendem a necessidades de informação para apoio à tomada de decisão de setores outros que não apenas aqueles ligados à manufatura: distribuição física, custos, recebimento fiscal, faturamento, recursos humanos, finanças, contabilidade, entre outros, todos integrados entre si e com os módulos de manufatura, a partir de uma base de dados única e não redundante.

Apesar de serem diferentes os enfoques dados pela literatura ao mencionar os Sistemas Integrados de Gestão, Valente (2004) afirma que, em geral, o que caracteriza um sistema ERP, diferenciando-o dos pacotes de softwares tradicionais, é a sua integração em tempo real e por possuir um banco de dados único.

\subsection{ERP nas pequenas e médias empresas}

A literatura sobre os ERP's direcionada a analisar estes sistemas em empresas de menor porte ainda é escassa e sem dúvida merece maiores pesquisas que possam nortear o caminho de empresas clientes e fornecedoras deste segmento e dos seus sistemas de informação (VALENTE, 2004; SUSSMANN, 2005).

Os sistemas ERP's foram inicialmente projetados para atender às grandes corporações, isto porque o seu custo de aquisição e implantação era muito elevado para as empresas de menor porte. Com o passar do tempo a maioria das grandes empresas já tinham seus sistemas integrados, logo os fornecedores se viram com o mercado reduzido para seus produtos. Foi quando designaram seu foco também para as empresas menores através de softwares com custos mais acessíveis (SUSSMANN, 2005; CARVALHO et al., 2009).

Apesar do alto custo dos softwares ser um empecilho para a implantação, outros fatores devem ser considerados. No estudo realizado por Snider, Silveira e

Revista Produção Online, Florianópolis, SC, v.14, n. 2, p. 533-559, abr./jun. 2014. 
Balakrishnan (2009), para verificar os fatores críticos de sucesso na implantação de ERP's nas PME's, foi verificado que as principais vantagens das PME's, quando comparado com grandes empresas, são: envolvimento diário da alta gerência e estrutura organizacional e cultura informal, que proporcionam agilidade na tomada de decisão.

Como desvantagens os autores destacam os seguintes pontos: falta de recursos humanos qualificados; ausência de planejamentos de longo prazo; falta de uma equipe ou departamento estruturado de TI e, finalmente, o custo de uma implementação de ERP pode ser proporcionalmente maior para as PME's do que para as grandes organizações, de modo que as PME's podem ter mais dificuldades para realizarem implementações de sucesso.

Mesmo com algumas desvantagens, comparado com as grandes empresas, as PME's começaram a sentir a necessidade de sistemas de informação que pudessem proporcionar maior competitividade em seus ambientes de negócio.

A oferta acessível de tecnologias de informação e a dependência cada vez maior das empresas por informações proporcionaram uma corrida para se possuir sistemas cada vez mais sofisticados, de modo a suportar as atividades internas. Assim, a informação tornou-se uma ferramenta poderosa de competitividade entre as empresas (ALVARENGA, 2003).

Sobre a característica da falta de recursos nas PME's, Mendes e Escrivão Filho (2002, p.290) afirmam que "a implantação de ERP nas PME's é um fato que gera preocupação e cuidados, pois esse segmento, em geral, não possui muitos recursos disponíveis para investimentos em tecnologia." Diante disto, os preços dos sistemas ERP acabam sendo o primeiro critério na hora de selecioná-los. Por isso, Mendes e Escrivão Filho (2002 apud VALENTE, 2004, p. 66) alertam para

[...] o fato de que nesse segmento é preciso haver confiabilidade no fornecedor, pois muitas empresas fornecedoras de sistema ERP são relativamente novas e também de pequeno porte, oferecendo, em razão disso, produtos com preços atraentes para o segmento das PME's.

Mendes e Escrivão Filho (2002) também destacam que nas PME's, a verificação da real necessidade do ERP nem sempre é investigada a fundo, trazendo, com isso, altos investimentos com retornos não compensáveis. Os mesmos autores também mencionam que, muitas vezes, outras soluções, por

Revista Produção Online, Florianópolis, SC, v.14, n. 2, p. 533-559, abr./jun. 2014. 
exemplo, a revisão de processos, seriam suficientes para atingir objetivos das PME's, mas elas acabam optando pela implantação do ERP, que é uma opção muito mais dispendiosa e nem acabam utilizando o potencial que o software oferece.

Diante das características apresentadas, percebe-se que há profundas diferenças entre as empresas de pequeno e médio porte com relação às grandes empresas no que tange aos sistemas integrados de gestão.

\subsection{Usuários dos sistemas ERP's}

O fator humano é apresentado na literatura como um dos principais recursos influenciadores no sucesso de funcionamento de um sistema de informação. Muitas das implantações de ERP's têm fracassado devido à ênfase dada unicamente ao recurso tecnológico oferecido pelo software, em contraposição ao esquecimento da importância do fator humano, tanto com relação aos usuários como também com a parte gerencial (SUSSMANN, 2005).

\subsubsection{Tipos de usuários dos ERP's}

Um sistema de informação pode possuir diversos tipos de usuários dependendo de suas habilidades, conhecimentos e formas de atuação. Nos sistemas ERP's alguns autores enfatizam dois principais tipos de usuários: os usuários-chave e os usuários-finais (WU; WANG, 2007, SILVA, 2003) Este trabalho possui como ênfase a análise destes dois tipos de usuários e os mesmos podem ser definidos como:

- Usuários-chave: Autores como Schmitt (2004), Wu e Wang (2007) descrevem que este tipo de usuário é geralmente selecionado pelos implementadores dos softwares ERP's dentre os colaboradores mais familiarizados com os processos da organização nos departamentos em que atuam, pela autonomia funcional e pela liderança. Eles acabam auxiliando os desenvolvedores do sistema e também se especializam em determinadas partes do sistema para atuarem como multiplicadores deste conhecimento.

Revista Produção Online, Florianópolis, SC, v.14, n. 2, p. 533-559, abr./jun. 2014. 
- Usuários-finais: O' Brien (2004) define o usuário final como sendo qualquer pessoa que utilize um sistema de informação ou a informação que ele produz.

Em contraste com usuários-chave, os usuários-finais do sistema ERP possuem apenas um conhecimento muito específico das partes do sistema que necessitam para seu trabalho (WU; WANG, 2007).

\subsection{Avaliação da satisfação dos usuários dos sistemas ERP's}

É muito importante para uma organização avaliar o sucesso de implantação de uma nova tecnologia de informação, visto que muitos recursos financeiros e humanos são investidos (CHIEN; TSAUR, 2007 apud ELY, 2010). Este princípio torna-se ainda mais importante em empresas cujos recursos necessários para a implantação da tecnologia são escassos, como ocorre nas PME's.

A medição do impacto ocasionado na implantação de um software ERP através de uma análise direta dos benefícios com custos, produtividade, vantagem de competitividade e auxílio na tomada de decisões seria ideal. Porém, em virtude da dificuldade da medição destes parâmetros, a medição da satisfação dos usuários tem recebido uma aceitação generalizada como um substituto de medição de tais indicadores (WU et al., 2002; WU; WANG, 2007).

Por essa razão, acadêmicos e profissionais admitem que o sucesso de um sistema de informação pode ser avaliado diante da mensuração da satisfação de seu usuário (IVES; OLSON e BAROUD, 1983; DOLL; TORKZADEH, 1988; WU; WANG, 2007; ELY, 2010).

No decorrer dos anos surgiram diversos trabalhos com o objetivo de medir a satisfação dos usuários, tais trabalhos acompanharam o desenvolvimento das tecnologias desde os mainframes até os sistemas ERP.

O modelo de Ely (2010) apresenta 13 variáveis para os usuários finais divididos em 3 construtos (produto ERP, serviços dos usuários-chave e conhecimento e envolvimento); e 14 variáveis para o usuário chave, também divididos em 3 construtos (produto ERP, serviços dos consultores e conhecimento e 
envolvimento). A Figura 2 apresenta o modelo desenvolvido pela autora, o qual foi utilizado como base de pesquisa para esta pesquisa.

Figura 2 - Modelo de Pesquisa

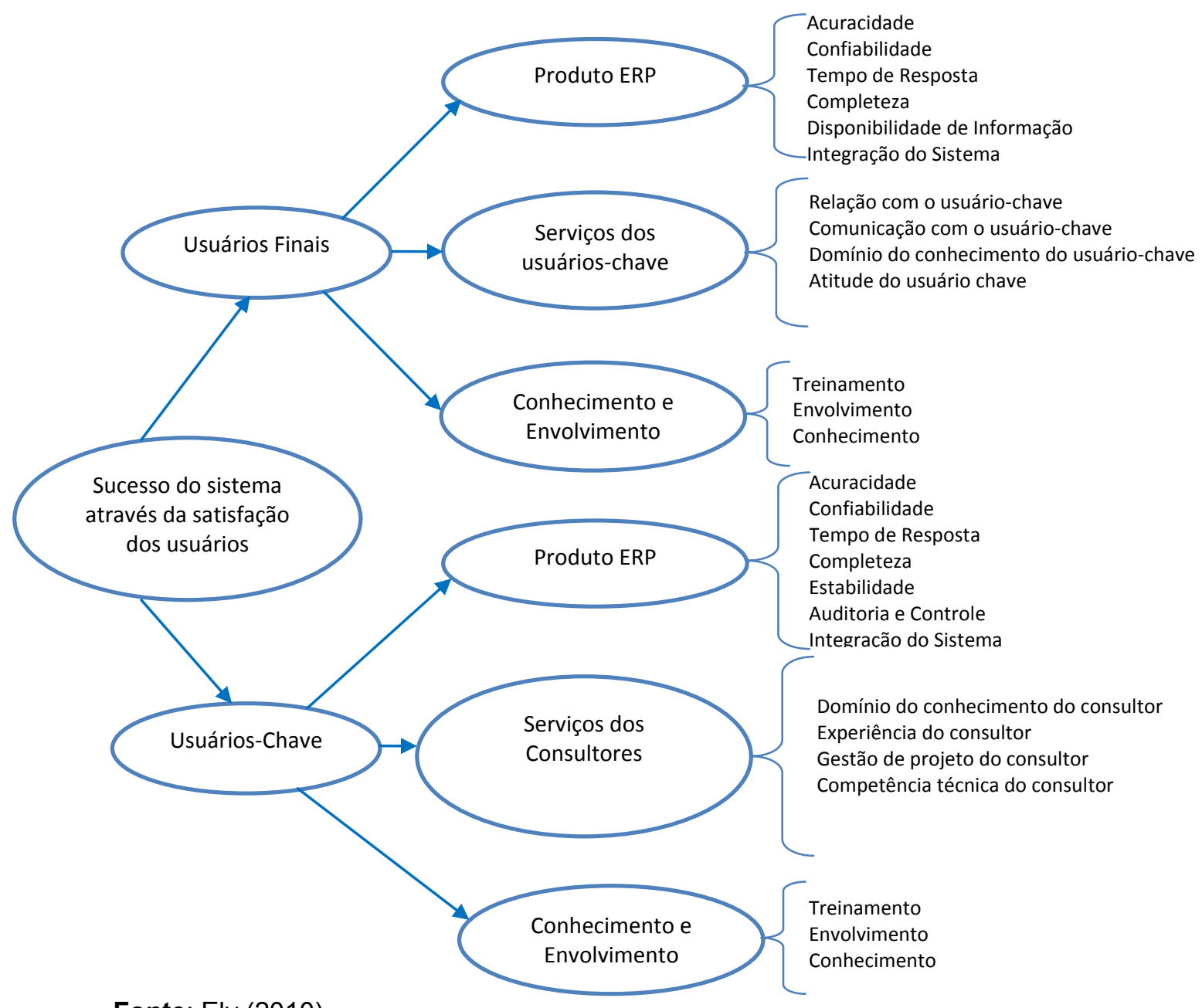

Fonte: Ely (2010)

Nesta pesquisa, o processo de observação do sucesso operacional dos sistemas ERP's nas ME's do município de Fortaleza foi realizado através da avaliação da satisfação dos usuários finais e chave do software ERP.

\section{PROCEDIMENTOS METODOLÓGICOS}

\subsection{Método de pesquisa}

Revista Produção Online, Florianópolis, SC, v.14, n. 2, p. 533-559, abr./jun. 2014. 
O método utilizado neste trabalho é a pesquisa survey, a qual pode ser descrita como a obtenção de dados ou informações sobre características, ações ou opiniões de determinado grupo de pessoas, indicados como representantes de uma população-alvo, por meio de um instrumento de pesquisa (PINSONNEAULT; KRAEMER, 1993). O presente trabalho trata-se de uma pesquisa survey porque avalia, de forma quantitativa e através de inferência estatística, a satisfação dos usuários finais e usuários-chave de sistemas ERP.

Poucos foram os trabalhos realizados no Brasil para medir especificamente a satisfação dos usuários de sistemas ERP's na fase pós-implantação. Desta forma, foi desenvolvido por Ely (2010) um trabalho com o intuito de reunir e customizar dois dos principais modelos de avaliação da satisfação dos usuários (usuários finais e usuários-chave) e testá-los e validá-los no contexto brasileiro. O modelo de Ely (2010) foi utilizado nesta pesquisa utilizando o segmento das médias empresas.

Neste trabalho estão configurados como instrumento de pesquisa três modelos de questionários: i) um para coletar informações das empresas alvo desta pesquisa e de seus respectivos sistemas ERP's; ii) um segundo para obter informações dos usuários finais e avaliar sua satisfação com relação ao sistema; iii) e o terceiro para obter informações dos usuários-chave e avaliar sua satisfação com relação ao sistema.

Os três questionários foram elaborados mesclando-se questões objetivas, subjetivas e com escalas tipo Likert de 1 a 7 , sendo 1 a pior avaliação, 4 neutro e 7 a melhor avaliação, ou seja, valores entre 1 e 3 apresentam uma avaliação negativa da satisfação do usuário com relação à variável, 4 indica uma posição neutra e valores entre 5 e 7 apresentam uma avaliação positiva da satisfação do usuário com relação à variável.

Foram descartados os questionários quando os números de questões respondidas tiveram menos que $50 \%$ de respostas, assim, todas as suas avaliações foram eliminadas do cálculo. Esta medida assegurou que os julgamentos de um determinado avaliador não sejam descartados indevidamente e que seu aproveitamento não seja prejudicial para a estimativa da confiabilidade. 
O intuito da pesquisa foi analisar as médias empresas de confecção do vestuário do município de Fortaleza que possuem um sistema ERP implantado há pelo menos 6 meses.

O critério para a seleção do porte destas empresas foi o utilizado pelo SEBRAE com parâmetros referentes ao número de funcionários, sendo de 100 a 499 funcionários para as médias empresas.

A relação das empresas pesquisadas foi obtida por meio do Guia Industrial do Ceará, elaborado pela Federação das Indústrias do Estado do Ceará - FIEC (2010), que apresenta um universo de 38 empresas de confecção do vestuário de médio porte, com um total de aproximadamente 6.500 colaboradores. Todas as 38 empresas fizeram parte da amostra da pesquisa, porém, apenas 25 empresas participaram da pesquisa, pois possuíam o sistema implantado há pelo menos 6 meses e/ou concordaram em responder os questionários.

Foram determinados para amostra final, os usuários com experiência mínima de seis meses no sistema ERP. O número de usuários com este perfil foi obtido após a coleta de dados do primeiro questionário. A quantidade de usuários das 25 empresas com o perfil estabelecido está relacionada na Tabela 1.

Tabela 1 - Universo da pesquisa

\begin{tabular}{lccc}
\hline UNIVERSO PESQUISA & $\begin{array}{c}\text { USUÁRIO } \\
\text { FINAL }\end{array}$ & $\begin{array}{c}\text { USUÁRIO- } \\
\text { CHAVE }\end{array}$ & TOTAL \\
\hline \hline Usuários de sistemas ERP & 966 & 133 & 1099 \\
\hline
\end{tabular}

A implementação da pesquisa consistiu na coleta direta dos dados, sendo realizada no período entre dezembro de 2011 e fevereiro de 2012, e a mesma está dividida em três etapas, ressaltadas no Quadro 1: 
Quadro 1 - Etapas de coleta de dados

\begin{tabular}{|c|c|}
\hline $\begin{array}{c}1^{\mathrm{a}} \\
\text { etapa }\end{array}$ & $\begin{array}{l}\text { Consistiu em um primeiro contato por meio de visita direta do pesquisador às } \\
\text { empresas foco. Neste primeiro contato procurou-se informar os objetivos da } \\
\text { pesquisa, apresentar resumidamente as informações necessárias e saber se a } \\
\text { empresa possui um software ERP implementado há no mínimo seis meses. } \\
\text { Caso a resposta tenha sido positiva, o empresário foi convidado a participar da } \\
\text { pesquisa. Em caso de aceite, iniciou-se a implementação da pesquisa. }\end{array}$ \\
\hline $\begin{array}{c}2^{\mathrm{a}} \\
\text { etapa }\end{array}$ & $\begin{array}{l}\text { Após o aceite, foi solicitada a indicação da pessoa responsável pelo setor de } \\
\text { TI que pudesse responder questões sobre a empresa e sobre o software ERP, } \\
\text { e que também pudesse acompanhar nas demais etapas da pesquisa. Este } \\
\text { primeiro questionário foi apresentado por meio impresso diretamente ao } \\
\text { respondente. }\end{array}$ \\
\hline $\begin{array}{c}3^{\mathbf{a}} \\
\text { etapa }\end{array}$ & $\begin{array}{l}\text { Com as respostas do primeiro questionário foi possível saber a quantidade de } \\
\text { usuários finais e de usuários-chave, os quais foram os respondentes do } \\
\text { segundo e do terceiro questionários. Então, a coleta de dados foi realizada } \\
\text { com a presença do pesquisador com documento impresso em uma única visita } \\
\text { à empresa (não foram deixados questionários para serem preenchidos em } \\
\text { outros momentos). Nesta etapa foi solicitada a participação de todos os } \\
\text { usuários que se encaixassem no perfil da amostra. }\end{array}$ \\
\hline
\end{tabular}

\subsection{Tratamento estatístico dos dados finais}

Alguns dados foram tratados através da estatística descritiva objetivando uma percepção global, outras variáveis que compõem a avaliação dos usuários receberam simultaneamente um tratamento estatístico através da técnica multivariada de Análise Fatorial (AF).

A AF é uma técnica multivariada de interdependência que objetiva estudar as inter-relações entre as variáveis em alguns fatores ocultos e inobserváveis. Sua utilização supõe que as variáveis podem ser agrupadas de acordo com suas correlações. Assim, obtém-se como resultado, grupos com todas as variáveis altamente correlacionadas entre si, mas com baixas correlações com variáveis de um grupo diferente (CORRAR; PAULO; DIAS FILHO, 2007; HAIR JUNIOR et al., 2005 apud SANTOS et al., 2011). A análise multivariada seguiu os passos conforme apresentado na Figura 3.

Revista Produção Online, Florianópolis, SC, v.14, n. 2, p. 533-559, abr./jun. 2014. 
Figura 3 - Passos de análise das variáveis.

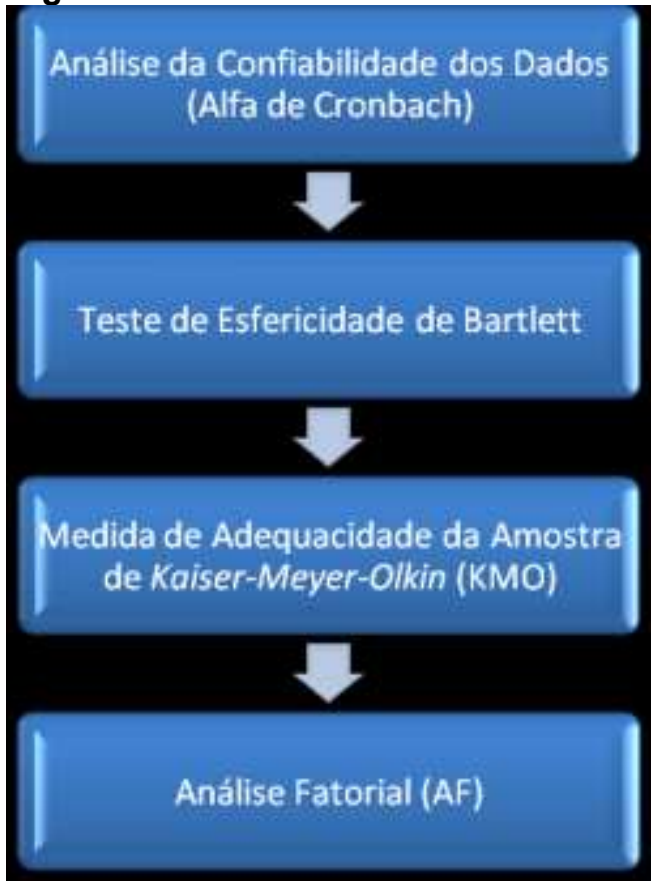

Fonte: Ely (2010)

\section{Análise da Confiabilidade dos Dados (Alfa de Cronbach)}

O Alfa de Cronbach é um dos modelos mais utilizados em análise de confiabilidade dos dados. É uma forma de estimar a confiabilidade de um questionário aplicado em uma pesquisa. O alfa mede a correlação entre respostas em um questionário através da análise do perfil das respostas dadas pelos respondentes, que permite determinar a extensão em que os itens estão relacionados entre si (CORRAR; PAULO; DIAS FILHO, 2007; HORA; MONTEIRO; ARICA, 2010).

Para verificar a consistência interna dos indicadores individuais da escala utilizada no instrumento de pesquisa deste trabalho, adotou-se como limite inferior de aceitabilidade o valor de 0,60 , ou seja, $60 \%$ representa o impacto real mínimo das variáveis.

\section{Teste de Esfericidade de Bartlett}

O Teste de Esfericidade de Bartlett avalia a presença de correlações entre as variáveis indicando se a análise fatorial é apropriada (ELY, 2010).

Revista Produção Online, Florianópolis, SC, v.14, n. 2, p. 533-559, abr./jun. 2014. 
Para que seja possível a aplicação da AF, recomenda-se que o valor de Sig. (teste de significância) não seja superior a 0,05 (CORRAR; PAULO; DIAS FILHO, 2007). Neste trabalho foi analisada, através da estatística qui-quadrado ao nível de 0,05 , a hipótese de que as variáveis no estudo não sejam correlacionadas na população.

\section{Medida de Adequacidade da Amostra de Kaiser-Meyer-Olkin (KMO)}

Este teste permite avaliar se os dados originais viabilizam a utilização da AF de forma satisfatória. Valores altos (entre 0,5 e 1,0) indicam que esse método é apropriado (SANTOS et al., 2011). Para Hair Jr. et al. (2005), a medida pode ser interpretada como segue: 0,80 ou acima, admirável; maior ou igual a 0,70 e abaixo de 0,80 , mediano; maior ou igual a 0,60 e abaixo de 0,70 , medíocre; maior ou igual a 0,50 e abaixo de 0,60, ruim; e abaixo de 0,50, inaceitável.

São adotados como aceitáveis valores entre 0,60 e 1,00. Os dados foram analisados com o software SPSS ${ }^{\circ}$ (v. 19). Os resultados favoráveis dos testes de Bartlett e KMO foram requisitos obrigatórios para a realização da Análise Fatorial nesta pesquisa.

\section{Análise Fatorial (AF)}

A Análise Fatorial pode ser utilizada para diversas finalidades, como por exemplo: redução do número de variáveis a serem consideradas em uma pesquisa, sumarização de dados permitindo a escolha de uma ou mais variáveis significativas para serem objeto de avaliação e acompanhamento, dentre outros (CORRAR; PAULO; DIAS FILHO, 2007).

O número de fatores é determinado utilizando-se a análise de componentes principais, que é um dos métodos multivariados mais simples, adotou-se como limite inferior de aceitabilidade das cargas fatoriais o valor de 0,60. A rotação utilizada neste estudo é a Varimax.

Nesta pesquisa a AF tem o objetivo de verificar o agrupamento das variáveis em seus respectivos fatores e tentar interpretar os possíveis motivos destes 
agrupamentos, assim como identificar as variáveis com baixas cargas fatoriais passíveis de exclusão. Nesta etapa também foi utilizado o softwareSPSS® (v. 19).

\section{RESULTADOS}

Durante a coleta de dados, quando foi consultado se a empresa possuía um sistema ERP, todas as 38 empresas do universo da pesquisa afirmaram possuir o software em operação ou em fase de implantação. No entanto, maiores detalhes sobre o sistema foram obtidos apenas com as 25 empresas que concordaram em participar da pesquisa e que estavam com o software em funcionamento há pelo menos 6 meses. Isto demonstra que, atualmente, este tipo de software está acessível e é considerado importante para as empresas de médio porte do segmento analisado.

A Tabela 2 apresenta o nome dos sistemas ERP's utilizados pelas 25 empresas consultadas. Os dois principais softwares, com 48\% de utilização, são o ALPHA e o GERA3. Também se verifica nesta tabela que há uma grande variedade de opções (10 fornecedores) de software para este segmento da confecção do vestuário.

Tabela 2 - ERP's utilizados pelas empresas

\begin{tabular}{lcc}
\hline \multicolumn{1}{c}{ ERP } & EMPRESAS & TAXA \\
\hline \hline ALPHA & 6 & $24 \%$ \\
GERA 3 & 6 & $24 \%$ \\
TAYLOR & 4 & $16 \%$ \\
MILLENIUM & 3 & $12 \%$ \\
GEPROD & 1 & $4 \%$ \\
IVI COM & 1 & $4 \%$ \\
LINOX & 1 & $4 \%$ \\
STORE_AGE & 1 & $4 \%$ \\
TOP MANAGER & 1 & $4 \%$ \\
UNUM & 1 & $4 \%$ \\
\hline \multicolumn{1}{c}{ Total } & $\mathbf{2 5}$ & $\mathbf{1 0 0} \%$ \\
\hline \hline
\end{tabular}

\subsection{Análises usuários final}

\subsubsection{Testes de análise multivariada (questionário usuário final)}


A avaliação por meio do Alfa de Cronbach indicou uma alta confiabilidade tendo em vista que o resultado foi $\boldsymbol{\alpha}=\mathbf{0 , 9 2 0}$ e o valor mínimo aceitável nesta pesquisa foi de $\alpha=0,600$.

$\mathrm{Na}$ análise dos resultados para os usuários finais foi obtido o valor de Sig.= 0,000, indicando que a AF é apropriada.

$\mathrm{O}$ teste KMO também confirmou a utilização da AF apresentando um valor de $\mathrm{KMO}=\mathbf{0 , 8 7 7}$, o que representa um bom grau de ajuste ao uso do método.

\subsubsection{Análise fatorial (usuário final)}

Após a obtenção de resultados satisfatórios com a realização dos testes de utilização da Análise Fatorial, foram estabelecidos os fatores utilizando todas as 13 variáveis de avaliação dos usuários finais simultaneamente. Tais indicadores formaram 3 fatores conforme apresentado na Tabela 3.

Tabela 3 - Análise fatorial usuário final.

\begin{tabular}{l|ccc}
\hline \multicolumn{1}{c}{ INDICADORES } & \multicolumn{3}{c}{ FATORES } \\
\hline \hline Completeza & $\mathbf{1}$ & $\mathbf{2}$ & $\mathbf{3}$ \\
Confiabilidade &, 894 &, 185 &, 131 \\
Integração &, 851 &, 209 &, 215 \\
Disponib. de informação &, 841 &, 176 &, 101 \\
Tempo de resposta &, 827 &, 216 &, 292 \\
Acuracidade &, 801 &, 169 &, 076 \\
Treinamento &, 795 &, 150 &, 202 \\
\hline Relação &, 610 &, 122 &, 279 \\
Domínio do conhecimento &, 059 &, 907 &, 145 \\
Comunicação &, 144 &, 865 &, 251 \\
Atitude &, 303 &, 839 &, 076 \\
\hline Conhecimento &, 317 &, 809 &, 122 \\
Envolvimento &, 229 &, 241 &, 833 \\
\hline
\end{tabular}

Os resultados das cargas fatoriais formaram 3 fatores bem distintos sendo que cada fator é composto pelas seguintes variáveis:

- Fator 1 (7 variáveis): Completeza; Confiabilidade; Integração; Disponibilidade de informação; Tempo de resposta; Acuracidade e Treinamento. 
- Fator 2 (4 variáveis): Relação; Domínio do conhecimento; Comunicação e Atitude.

- Fator 3 (2 variáveis): Conhecimento e Envolvimento.

Estes 3 fatores acabaram confirmando quase que completamente a estrutura do instrumento de pesquisa que divide as variáveis em 3 seções (construtos), sendo a primeira seção que avalia o Produto Sistema ERP, podendo ser esta a nomenclatura para o fator1; a segunda seção que é uma auto avaliação de Desempenho do Usuário final, podendo ser esta a nomenclatura para o fator 3 e a terceira seção que avalia as Habilidades do Usuário-chave, podendo ser esta a nomenclatura para o fator 2.

A única e surpreendente alteração com relação à estrutura do instrumento de pesquisa desenvolvido por Ely (2010) foi a agregação do indicador Treinamento junto ao fator 1, denominado Produto Sistema ERP. Este resultado demonstra que o treinamento está intimamente relacionado com a avaliação de satisfação que o usuário final percebe do Produto Sistema ERP, ou seja, o nível de satisfação do usuário final com relação ao Treinamento está bem mais relacionado com o Produto Sistema ERP do que com as Habilidades do Usuário-chave ou do próprio Desempenho do Usuário final. Isso mostra, talvez, que o treinamento de sistemas ERP deve ser mais uma característica intrínseca do produto, do que do próprio usuário. A forma de agregação dos indicadores para cada fator está representada na Figura 4.

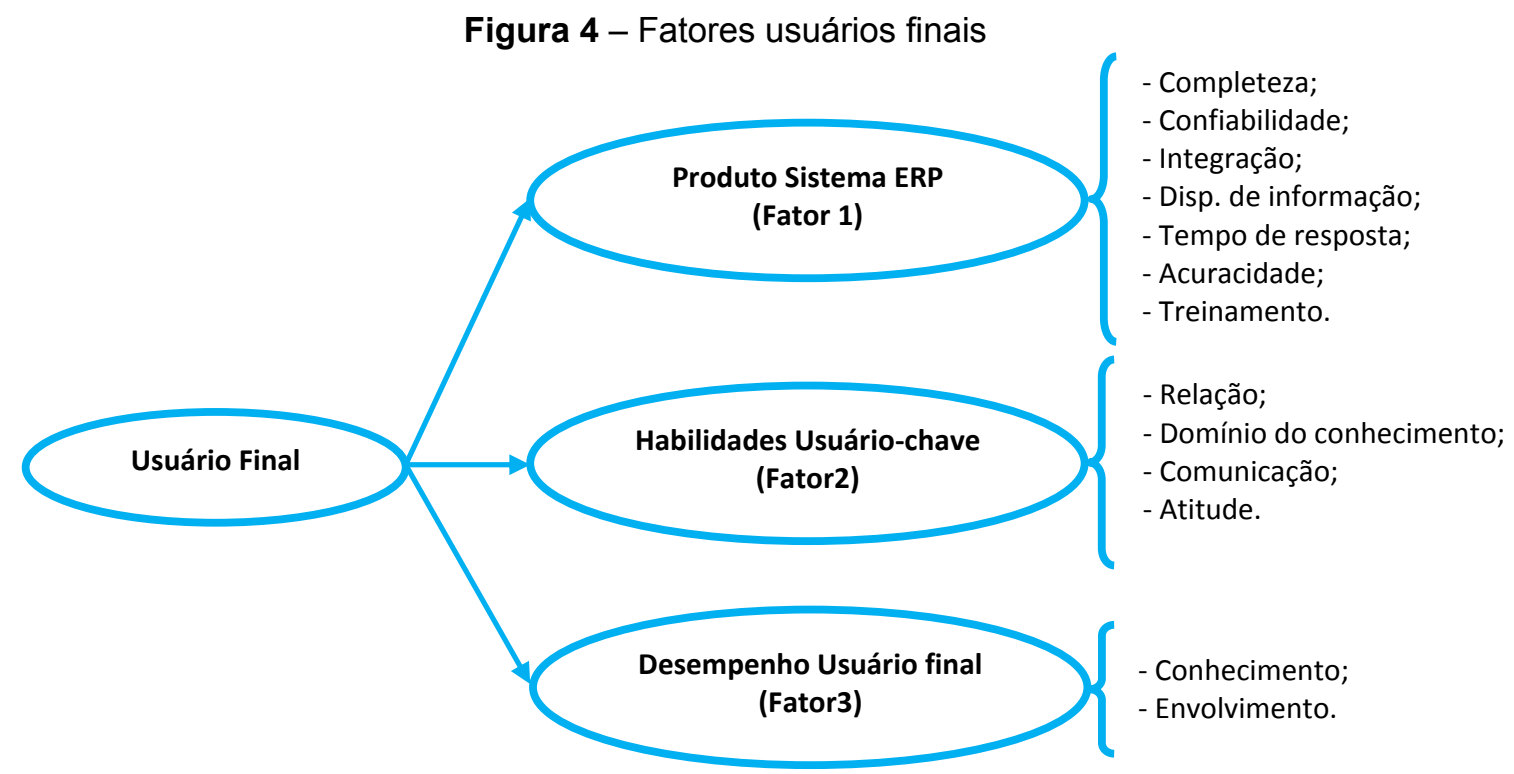

Revista Produção Online, Florianópolis, SC, v.14, n. 2, p. 533-559, abr./jun. 2014. 


\subsubsection{Análise das médias das variáveis (usuário final)}

A média e o desvio padrão das respostas obtidas sobre as variáveis que compõem a avaliação do usuário final estão apresentadas na Tabela 4. As variáveis que envolvem as questões relacionadas ao Produto ERP e ao Conhecimento e Envolvimento tiveram 88 respondentes e as variáveis que envolvem as questões relacionadas ao Usuário-chave, tiveram 78 respondentes.

Tabela 4 - Média das variáveis de satisfação do usuário final.

\begin{tabular}{lccc}
\multicolumn{1}{c}{ VARIÁVEL } & RESPONDENTES & MÉDIA & $\begin{array}{c}\text { DESVIO } \\
\text { PADRÃO }\end{array}$ \\
\hline \hline Acuracidade & 88 & 5,20 & 1,23 \\
Confiabilidade & 88 & 5,16 & 1,42 \\
Tempo de resposta & 88 & 5,08 & 1,38 \\
Completeza & 88 & 5,24 & 1,30 \\
Disponibilidade de informação & 88 & 5,26 & 1,29 \\
Integração & 88 & 5,00 & 1,39 \\
Envolvimento & 88 & 5,61 & 1,25 \\
Conhecimento & 88 & 5,17 & 1,36 \\
Treinamento & 88 & 4,67 & 1,60 \\
Relação & 78 & 5,91 & 1,34 \\
Comunicação & 78 & 5,94 & 1,46 \\
Domínio do conhecimento & 78 & 5,88 & 1,34 \\
Atitude & 78 & 5,79 & 1,54 \\
\hline \multicolumn{2}{c}{ MÉDIA GERAL } & $\mathbf{5 , 3 8}$ & \\
\hline
\end{tabular}

A média geral obtida na pesquisa $(5,38)$ apresentou que os usuários finais possuem uma avaliação positiva sobre o software ERP das empresas pesquisadas, embora seja um valor mais próximo de uma avaliação neutra (4) do que de uma melhor avaliação (7). Também se observa, pelo baixo valor dos desvios padrões encontrados, que as respostas foram bastante homogêneas.

As médias das variáveis encontradas estão entre 4,67 e 5,94, sendo que a variável treinamento foi a que apresentou o menor resultado e a variável comunicação a que apresentou o maior resultado.

\subsection{Análises usuário-chave}

\subsubsection{Testes de análise multivariada (questionário usuário-chave)}

Revista Produção Online, Florianópolis, SC, v.14, n. 2, p. 533-559, abr./jun. 2014. 
A avaliação por meio do Alfa de Cronbach também indicou uma alta confiabilidade para os usuários-chave, tendo em vista que o resultado foi $\boldsymbol{\alpha}=\mathbf{0 , 9 3 9} \mathrm{e}$ o valor mínimo aceitável nesta pesquisa foi de $\alpha=0,600$.

No Teste de Esfericidade de Bartlett para os dados do usuário-chave houve uma boa indicação da existência de relação entre as variáveis para aplicação da Análise Fatorial. $\mathrm{Na}$ análise dos resultados foi obtido o valor de Sig. $\mathbf{0 , 0 0 0}$, ficando de acordo com o valor máximo estabelecido de 0,05 e indicando que a AF é apropriada.

O teste KMO também confirmou a utilização da AF, apresentando um valor de $\mathrm{KMO}=\mathbf{0 , 7 8 7}$.

\subsubsection{Análise fatorial (usuário-chave)}

Após a obtenção de resultados satisfatórios com a realização dos testes de utilização da Análise Fatorial, foram estabelecidos os fatores utilizando todas as 14 variáveis de avaliação dos usuários-chave simultaneamente. Tais indicadores formaram apenas 2 fatores e com a aplicação da rotação dos fatores pelo critério Varimax, foi possível organizá-los conforme apresentado na Tabela 5.

Tabela 5 - Análise fatorial usuário-chave.

\begin{tabular}{l|cc}
\hline \multicolumn{1}{c|}{ INDICADORES } & \multicolumn{2}{c}{ FATORES } \\
& $\mathbf{1}$ & $\mathbf{2}$ \\
\hline \hline Estabilidade &, 839 &, 234 \\
Acuracidade &, 825 &, 361 \\
Confiabilidade &, 809 &, 258 \\
Completeza &, 807 &, 178 \\
Tempo de resposta &, 805 &, 113 \\
Integração &, 677 &, 518 \\
Auditoria &, 666 &, 241 \\
Envolvimento &, 583 &, 565 \\
\hline Experiência &, 270 &, 873 \\
Domínio do conhecimento &, 270 &, 804 \\
Conhecimento &, 337 &, 763 \\
Treinamento &,- 026 &, 719 \\
Gestão de projeto &, 514 &, 698 \\
Comp. Técnica &, 471 &, 686 \\
\hline
\end{tabular}

Revista Produção Online, Florianópolis, SC, v.14, n. 2, p. 533-559, abr./jun. 2014. 
Diferente do que ocorreu na avaliação dos usuários finais em que os fatores seguiram a estrutura do instrumento de pesquisa, com seus 3 construtos, na avaliação dos usuários-chave a organização das cargas fatoriais ficou dividida em apenas 2 fatores, sendo eles:

- Fator 1 (8 variáveis): Estabilidade; Acuracidade; Confiabilidade; Completeza; Tempo de resposta; Integração; Auditoria e Envolvimento.

- Fator 2 (6 variáveis): Experiência; Domínio do conhecimento; Conhecimento; Treinamento; Gestão de projeto e Competência técnica.

O fator 1 ficou agrupado com todas as variáveis que avaliam a satisfação do usuário-chave com o Produto Sistema ERP, assim como estava organizado no primeiro construto do instrumento de pesquisa, e assim como ocorreu com a AF do usuário final.

O único indicador agrupado neste fator proveniente de outra seção do instrumento de pesquisa foi o Envolvimento, o qual se refere ao envolvimento do usuário-chave com o ERP. Apesar deste indicador estar agrupado no fator 1, percebe-se que o mesmo possui uma carga fatorial baixa $(<0,600)$ e que também apresenta uma carga bastante parecida no fator 2 , conforme verificado na Tabela 5 , ou seja, o indicador Envolvimento tanto é pouco relacionado com o fator 1 como com o fator 2, portanto, devido sua baixa carga fatorial o mesmo foi excluído, restando apenas 13 variáveis no total e 7 indicadores no fator 1 .

O fator 2 ficou agrupado com os indicadores que compõem o Desempenho do usuário-chave (segundo construto do instrumento de pesquisa), menos o indicador Envolvimento; e com todos os indicadores que avaliam as Habilidades do Consultor/Fornecedor (terceiro construto do instrumento de pesquisa). Portanto, a AF aponta que o Desempenho do usuário-chave está diretamente relacionado às Habilidades do Consultor/Fornecedor. Este fator 2 ficou denominado como Competências de gestão, pois tanto o usuário-chave como os consultores/fornecedores são os responsáveis pelo comando de operacionalização do software em toda a organização, ou seja, o treinamento e conhecimento do usuário-chave relaciona-se em alto grau com as competências do Consultor/Fornecedor. 
A forma de agregação dos indicadores para cada fator está representada na Figura 5.

Figura 5 - Fatores usuários-chave

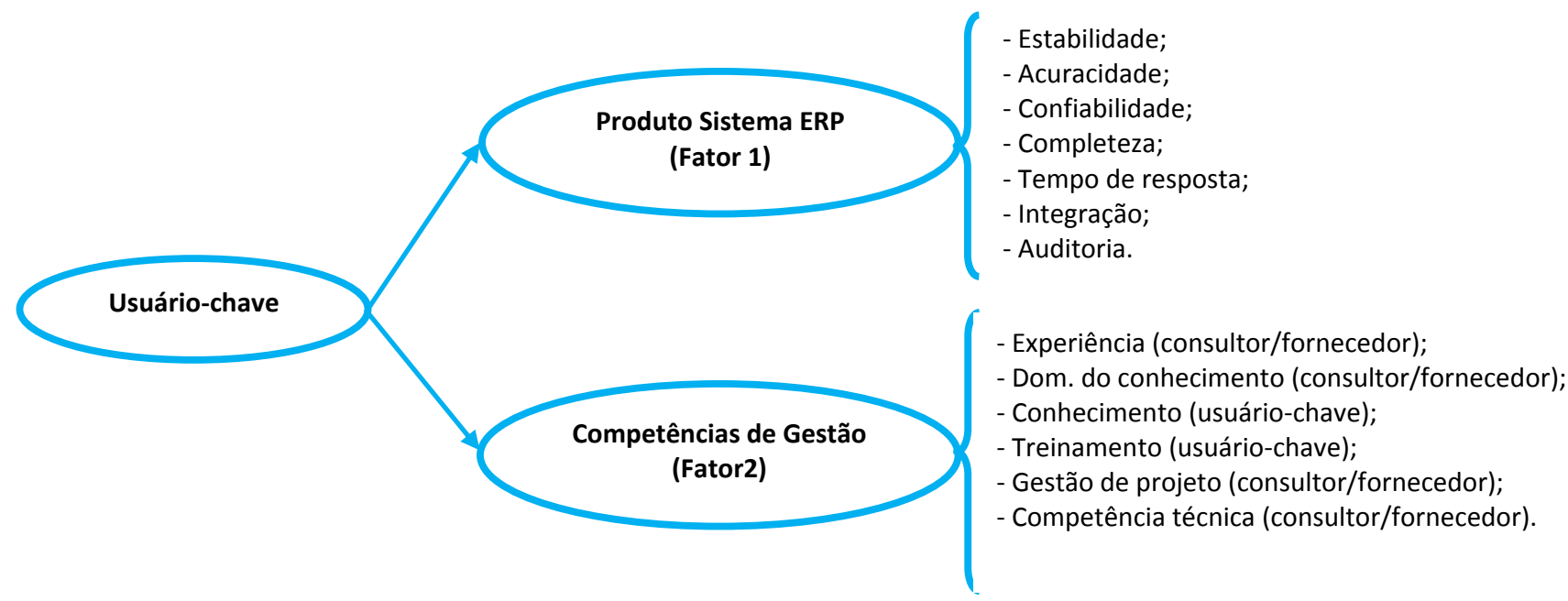

\subsubsection{Análise das médias das variáveis (usuário-chave)}

A média e o desvio padrão das respostas obtidas sobre as variáveis que compõem a avaliação do usuário-chave estão apresentadas na Tabela 6. Cada variável obteve 29 respondentes.

Tabela 6 - Média das variáveis de satisfação do usuário-chave.

\begin{tabular}{lccc}
\multicolumn{1}{c}{ Variável } & Respondentes & Média & $\begin{array}{c}\text { Desvio } \\
\text { Padrão }\end{array}$ \\
\hline \hline Confiabilidade & 29 & 5,28 & 1,51 \\
Tempo de resposta & 29 & 5,14 & 1,66 \\
Completeza & 29 & 5,17 & 1,44 \\
Estabilidade & 29 & 5,52 & 1,40 \\
Auditoria & 29 & 5,21 & 1,47 \\
Integração & 29 & 5,03 & 1,18 \\
Envolvimento & 29 & 5,97 & 1,40 \\
Conhecimento & 29 & 5,62 & 1,35 \\
Treinamento & 29 & 5,41 & 1,38 \\
Domínio do conhecimento & 29 & 5,38 & 1,40 \\
Experiência & 29 & 5,10 & 1,47 \\
Gestão do projeto & 29 & 5,00 & 1,28 \\
Competência técnica & 29 & 5,41 & 1,24 \\
\hline \multicolumn{1}{c}{ MÉDIA GERAL } & $\mathbf{5 , 3 3}$ & \\
\hline
\end{tabular}

Revista Produção Online, Florianópolis, SC, v.14, n. 2, p. 533-559, abr./jun. 2014. 
A média geral obtida na pesquisa $(5,33)$ apresentou que os usuários-chave possuem uma avaliação positiva sobre o software ERP das empresas pesquisadas, embora seja um valor mais próximo de uma avaliação neutra (4) do que de uma melhor avaliação (7). Também se observa, pelo baixo valor dos desvios padrões encontrados, que as respostas foram bastante homogêneas.

As médias das variáveis encontradas estão entre 5,00 e 5,97, sendo que a variável gestão do projeto foi a que apresentou o menor resultado e a variável envolvimento a que apresentou o maior resultado.

\section{CONSIDERAÇÕES FINAIS}

Embora a literatura apresente algumas dificuldades na implantação dos sistemas ERP's por empresas de pequeno e médio porte, pelo menos as empresas de médio porte do setor analisado estão conseguindo atingir níveis satisfatórios de avaliação.

$\mathrm{Na}$ análise da média das variáveis, os usuários finais, de um modo geral, avaliaram satisfatoriamente o sistema (média $=5,38$ ). Os resultados foram bastante homogêneos, conforme observado nos desvios padrões que variaram entre 1,23 e 1,60. A variável treinamento obteve a menor média e como a análise fatorial aponta que $\mathrm{o}$ treinamento está fortemente ligado às características do produto, provavelmente, cabe aos fornecedores desenvolverem meios de melhorar os treinamentos e os suportes técnicos aos usuários finais;

$\mathrm{Na}$ análise da média das variáveis, os usuários-chave, de um modo geral, avaliaram satisfatoriamente o sistema (média $=5,33$ ) e não houve grandes diferenças entre as médias das variáveis, as quais ficaram entre 5,00 e 5,97. Os resultados foram bastante homogêneos, conforme observado nos desvios padrões, que variaram entre 1,24 e 1,66.

O objetivo avaliar a satisfação dos usuários de Sistemas Integrados de Gestão (ERP) em empresas de médio porte do segmento de confecções do vestuário no município de Fortaleza foi atingido, tendo em vista que foi analisada uma amostra representativa de empresas e com uma taxa de respostas que se enquadra satisfatoriamente em um método Survey.

Revista Produção Online, Florianópolis, SC, v.14, n. 2, p. 533-559, abr./jun. 2014. 
Com os resultados apresentados pode-se concluir que a utilização dos sistemas ERP's pelas empresas são considerados bem sucedidos, pois seus usuários avaliaram positivamente a satisfação com a utilização dos sistemas. A pesquisa também possibilitou uma análise desta satisfação de forma detalhada com a divisão das variáveis em fatores, os quais possibilitam verificar em quais pontos a utilização do sistema é mais bem avaliada.

\section{REFERÊNCIAS}

ALVARENGA, M. L. F. Metodologia para verificação do sucesso na implantação de ERP (Enterprise Resources Planning) baseada nos fatores críticos de sucesso - aplicação na indústria mineira. Pesquisa (Mestrado em Engenharia de Produção) - Programa de Pós-Graduação em Engenharia de Produção da Universidade Federal de Santa Catarina, Florianópolis, 2003.

BRASIL. Ministério do Desenvolvimento, Indústria e Comércio Exterior. A Microempresa e Empresa de Pequeno Porte. Disponível em:

http://www2.desenvolvimento.gov.br/sitio/sdp/proAcao/micEmpPequeno/micEmpPeq ueno.php . Acesso em: 11 ago. 2010a.

CARVALHO, R. B. et al. Fatores-chave na implantação de ERP's: estudo de um caso problemático em uma média indústria. Revista Eletrônica de Sistemas de Informação, v.8, n.2, artigo 7, 2009.

CORRAR, L. J.; PAULO, E.; DIAS FILHO, J. M. Análise multivariada:para os cursos de administração, ciências contábeis e economia. São Paulo: Atlas, 2007.

CORREAA, H. L.; GIANESI, I. G. N.; CAON, M. Planejamento, programação e controle da produção: MRP II / ERP: conceitos, uso e implantação. 4. ed. São Paulo: Atlas, 2001.

COSTA, A. C. R. da; ROCHA, E. R. P. da. Panorama da cadeia produtiva têxtil e de confecções e a questão da inovação. BNDES Setorial, Rio de Janeiro, n. 29, p. 159-202, mar. 2009.

DAVENPORT, T. H. Putting de enterpriseintotheenterprise system. Harvard Business Review.p. 1221-1231, jul./ago. 1998.

DELOITTE CONSULTING. As Pequenas e médias empresas que mais crescem no Brasil. Disponível em: http://www.deloitte.com/assets/DcomBrazil/Local\%20Assets/Documents/PesqExame2008.pdf . Acessoem: 12 ago. 2010.

DINIZ FILHO, A. Indústria têxtil e de confecção: emprego e renda para o Brasil. Disponível em:

Revista Produção Online, Florianópolis, SC, v.14, n. 2, p. 533-559, abr./jun. 2014. 
http://www.guiatextil.com/site/noticias/economia/industria_textil_e_de_confeccao_em prego_e_renda_para_o_brasil.Acessoem11 dez. 2011.

DOLL, W. J.; TORKZADHE, G.The measurement of end-user computing satisfaction.MIS Quarterly, Minneapolis, v. 12, n. 1, p. 259-274, 1988. http://dx.doi.org/10.2307/248851

ELY, P. B. Medindo a satisfação dos usuários finais e dos usuários chave de um sistema de gestão empresarial, na empresa Springer Carrier. Pesquisa (Mestrado em Administração) - Escola de Administração da Universidade Federal do Rio Grande do Sul, Porto Alegre, 2010.

ENNS, S.T. MRP performance effects due to lot size and planned lead time settings. International Journal of Production Research,v. 39, 461-480, 2001. http://dx.doi.org/10.1080/00207540010002810

FEDERAÇÃO DAS INDÚSTRIAS DO ESTADO DO CEARÁ - FIEC. Guia industrial 2010. Fortaleza: FIEC, 2010.

HAIR Jr., J. F. et al. Análise multivariada de dados. 5. ed. Porto Alegre: Bookman, 2005.

HORA, H. R. M. da.; MONTEIRO, G. T. R.; ARICA, J. Confiabilidade em questionários para qualidade: um estudo como coeficiente alfa de cronbach. Produto eProdução. Porto Alegre, v. 11, n. 2, p. 85 - 103, jun. 2010.

INTERNATIONAL DATA CORPORATION (IDC BRASIL). Mercado brasileiro de ERP cresce $17 \%$ apesar da crise. 2009. Disponível em:

$<$ http://www.idclatin.com/news.asp?ctr=braeyear=2010eid release $=1644>$. Acesso em: 17 fev. 2010.

IVES, B.; OLSON, M. H.; BAROUDI, J. J. The measurement of user information satisfaction. Communications of the ACM, New York, v. 26, n. 10, 785-793, 1983. http://dx.doi.org/10.1145/358413.358430

$\mathrm{KOH}$, S.C.L., SAAD, S.M., 2003. A holistic approach to diagnose uncertainty in ERPcontrolled manufacturing shop floor. International Journal of Production Planning and Control, v. 14, n.3, p. 273-289.

http://dx.doi.org/10.1080/0953728031000111487

LAUDON, K. C.; LAUDON, J. P. Sistemas de informações gerenciais:

administrando a empresa digital. 5. ed. São Paulo: Pearson, Prentice Hall, 2004.

LEMOS, A. Q. et al. Gerenciando os caminhos para mudança: um estudo sobre o ambiente interno de confecções cearenses. In: Rio de Janeiro. CONGRESSO NACIONAL DE ADMINISTRAÇÃO E CIÊNCIAS CONTÁBEIS, 2., 2011. Anais... Rio de Janeiro, 2011. 
MALHOTRAA, R.; TEMPONIB, C. Criticaldecisions for ERP integration: small business issues. International Journal of Information Management, v. 30, p. 2837, 2010. http://dx.doi.org/10.1016/j.ijinfomgt.2009.03.001

MENDES, J. V.; ESCRIVÃO FILHO, E. Sistemas integrados de gestão ERP em pequenas empresas: um confronto entre o referencial teórico e a prática empresarial. Revista Gestão e Produção, São Carlos, v.9, n.3, p. 277 - 296, dez. 2002.

MILTENBURG, J. Computational complexity of algorithms for MRP and JIT production planning problems in enterprise resource planning systems. International Journal of Production Planning and Control, v. 12, n. 2, p.198-209, 2001.

O' BRIEN, J. A. Sistemas de informação e as decisões gerenciais na era da internet. 2. ed. São Paulo: Saraiva, 2004.

OLIVEIRA, R. C. R.; CUNHA, M. X. C.; SOUZA JUNIOR, M. F. Participação dos usuários no desenvolvimento de sistemas de informação: uma reflexão sob a ótica positivista e interpretativista. Revista do IESP, v. 7, p. 147-171, 2008.

PINSONNEAULT, A.; KRAEMER, K. L. Survey research in management information systems: an assessment. Journalof Management Information System, v. 10, n. 2, p. 75-106, 1993.

PINTO NETO, J. A. A análise da pré-venda na comercialização/aquisição de sistemas ERP nas empresas de Fortaleza. Pesquisa (Mestrado em Administração) - Universidade Federal do Ceará, Fortaleza, 2007..

SANTOS, C. de L. M. et al.Fatores de estresse na atividade de médicos em João Pessoa. Produção, v. 21, n. 1, p. 181-189, jan./mar. 2011.

http://dx.doi.org/10.1590/S0103-65132011005000003

SCHMITT, C. A. Sistemas integrados de gestão empresarial: uma contribuição no estudo do comportamento organizacional e dos usuários na implantação de sistemas ERP.Tese (Doutorado) - Programa de Pós-Graduação em Engenharia de Produção da Universidade Federal de Santa Catarina, Florianópolis, 2004.

SILVA, S. V. Qualidade de software - uma abordagem baseada na satisfação do usuário.Dissertação (Mestrado) - Centro de Ciência e Tecnologia da Universidade Estadual do Norte Fluminense, Campos dos Goytacazes, 2003.

SNIDER, B.; SILVEIRA, G. J. C. da; BALAKRISHNAN, J. ERP implementation at SMEs: analysis of five Canadian cases. International Journal of Operations and Production Management, v. 29, n.1, p. 4-29. 2009.

http://dx.doi.org/10.1108/01443570910925343

SOUZA, C. A. de. Sistemas integrados de gestão empresarial: estudos de casos de implementação de sistemas ERP.Pesquisa (Mestrado) - Faculdade de 
Economia, Administração e Contabilidade da Universidade de São Paulo, São

Paulo, 2000.

STAIR, R. M.; REYNOLDS, G. W. Princípios de sistemas de informação: uma abordagem gerencial.6. ed. São Paulo: Thomson, 2006.

SUSSMANN, A. G. Panorama dos sistemas ERP's nas médias empresas da cadeia de suprimentos das indústrias de autopeças de uma cidade do interior de São Paulo.Pesquisa (Mestrado em Administração) - Departamento de Administração da Faculdade de Economia, Administração e Contabilidade da Universidade de São Paulo, São Paulo, 2005.

VALENTE, N. T. Z. Implementação de ERP em pequenas e médias empresas: estudo de caso em empresa do setor da construção civil.Pesquisa (Mestrado) Universidade de São Paulo, São Paulo, 2004.

WU, J.; WANG, Y. Measureing ERP sucess: the key-users viewpoint of the ERP to produce a viable is in the organization. Computers in Human Behavior. Japan, $v$. 23, n. 3, p. 1582-1596, may 2007.

WU, J. et al. An examination of ERP user satisfaction in Taiwan.Computers in Human Behavior. Proceedings of the 35th Hawaii International Conference on System Sciences, 2002.



Artigo recebido em 08/07/2012 e aceito para publicação em 12/05/2014

DOI: http://dx.doi.org/10.14488/1676-1901.v14i2.1374 